\title{
A thin disk model for the high efficiency jet in powerful lobe-dominated FRII radio galaxies
}

\section{Shuang-Liang Li}

Key Laboratory for Research in Galaxies and Cosmology, Shanghai Astronomical Observatory, Chinese Academy of Sciences, 80 Nandan Road, Shanghai 200030, China;

email: lisl@shao.ac.cn

\begin{abstract}
We propose a thin accretion disk with magnetically driven winds model to investigate the high jet efficiency phenomenon in lobe-dominated luminous FRII radio galaxies, which was reported by Fernandes et al. (2011) and Punsly (2011). It was found that the temperature of a thin disk with winds was much lower comparing with that of a standard thin disk because the winds take the most of energy released in the disk away, resulting in a much smaller raditive efficiency. Therefore, the jet efficiency can be very high even for conventional jet power. Our model can explain the observations quite well.
\end{abstract}

Keywords. accretion, accretion disks, galaxies: active, black hole physics, galaxies: magnetic fields.

\section{Introduction}

Radio galaxies can be divided into two classed (FRI and FRII) according to their lobe morphology. It was usually believed that FRI and FRII radio galaxies should have different accretion model due to their different accretion rates (Ghisellini \& Celotti 2001). A radiatively efficient accretion disk is suggested to be present in FRII radio galaxies. Punsly (2011) and Fernandes et al. (2011) reported that a maximum jet efficiency line $R=25\left(R=L_{\text {jet }} / L_{\mathrm{bol}}\right)$ exists in some lobe-dominated luminous FRII galaxies.

The bolometric luminosity of a thin disk can be expressed as $L_{\mathrm{bol}}=\eta_{\mathrm{th}} \dot{M} c^{2}$, where $\eta_{\mathrm{th}}$ is the radiative efficiency. Together with the jet power $\left(L_{\mathrm{jet}}=\eta_{\mathrm{Q}} \dot{M} c^{2}\right.$, here $\eta_{\mathrm{Q}}$ is the jet production efficiency), the jet efficiency $R\left(=L_{\mathrm{jet}} / L_{\mathrm{bol}}=\eta_{\mathrm{Q}} / \eta_{\mathrm{th}}\right)$ will be decided by both $\eta_{\mathrm{Q}}$ and $\eta_{\mathrm{th}}$. The jet production efficiency for a magnetically-arrested-disk (MAD) can reach $\sim 30 \%$ and $140 \%$ for $a=0.5$ and 0.99 , respectively (Tchekhovskoy et al. 2011). Therefore, the jet efficiency can reach about 14 for a thin disk surrounding Kerr black hole $\left(\eta_{\mathrm{th}}=0.1\right.$ is adopted). However, if the radiative efficiency $\eta_{\mathrm{th}}$ of a thin disk were much smaller than 0.1 , we can get a high jet efficiency $R$ even for conventional jet production efficiency $\eta_{\mathrm{Q}}$. As presented in Li (2014) and Li \& Begelman (2014), they do find the temperature of a thin disk with magnetically driven winds/jets can decrease more than one order of magnitude, which directly results in the decrease of $\eta_{\mathrm{th}}$ and the increase of jet efficiency $R$.

\section{Models}

We consider a thin disk with magnetically driven winds surrounding a spinning black hole. The continuity, radial momentum, angular momentum and energy equations of the 
disk are as follows:

$$
\begin{gathered}
\frac{d}{d r}\left(2 \pi \Delta^{1 / 2} \Sigma v_{\mathrm{r}}\right)+4 \pi r \dot{m}_{\mathrm{w}}=0, \\
\frac{\gamma_{\phi} A M}{r^{4} \Delta} \frac{\left(\Omega-\Omega_{\mathrm{k}}^{+}\right)\left(\Omega-\Omega_{\mathrm{k}}^{-}\right)}{\Omega_{\mathrm{k}}^{+} \Omega_{\mathrm{k}}^{-}}+g_{\mathrm{m}}=0, \\
-\frac{\dot{M}}{2 \pi} \frac{d L}{d r}+\frac{d}{d r}\left(r W_{\phi}^{r}\right)+T_{\mathrm{m}} r=0, \\
\nu \Sigma \frac{\gamma_{\phi}^{4} A^{2}}{r^{6}}\left(\frac{d \Omega}{d r}\right)^{2}=\frac{16 a c T^{4}}{3 \bar{\kappa} \Sigma},
\end{gathered}
$$

where the equations and the meaning of parameters are all the same as in Li (2014).

The bolometric luminosity of accretion disk can be calculated as

$$
L_{\mathrm{bol}}=\int_{r_{\mathrm{in}}}^{r_{\mathrm{out}}} Q_{\mathrm{rad}} 2 \pi r d r=\eta_{\mathrm{th}} \dot{M} c^{2} .
$$

Jets can be powered by both BZ (Blandford \& Znajek 1977) and BP (Blandford \& Payne 1982) processes. In the general form of the BZ process, the jet power $L_{\mathrm{BZ}}$ can be calculated with

$$
L_{\mathrm{BZ}}=\frac{1}{32} \omega_{\mathrm{F}}^{2} B_{\perp}^{2} r_{\mathrm{H}}^{2}\left(J / J_{\max }\right)^{2} c .
$$

The jet power from an accretion disk (BP process) can be given as in Cao (2002)

$$
L_{\mathrm{BP}}=\int_{r_{\text {in }}}^{r_{\text {out }}} \frac{B_{\mathrm{p}} B_{\phi}}{4 \pi} r \Omega 2 \pi r \mathrm{~d} r,
$$

where $B_{\phi}$ and $B_{\mathrm{p}}$ are the toroidal and poloidal components of magnetic field, respectively.

Therefore the total jet power $L_{\text {jet }}$ is:

$$
L_{\text {jet }}=L_{\mathrm{BZ}}+L_{\mathrm{BP}}=\eta_{\mathrm{Q}} \dot{M} c^{2}
$$

With $\eta_{\mathrm{th}}$ and $\eta_{\mathrm{Q}}$, the jet efficiency is given by:

$$
R=\frac{\eta_{\mathrm{Q}}}{\eta_{\mathrm{th}}} .
$$

\section{Results And Discussion}

Previous studies mainly focused on the improvement of jet power. However, the strong large-scale magnetic field threading on the disk can not only improve jet power, but also decrease the radiative efficiency (Tchekhovskoy et al. 2011). We suggest that in order to explain the high jet efficiency in luminous FRII radio galaxies, the jet power is not necessary very high if the radiative efficiency is low. This can be realized because the winds/jets can take away most of gravitational energy released in the disk (see figure 1). A theoretical maximum jet efficiency $R \sim 1000$ is found, which is large enough even taking the episodic activity of jets into account.

The key point of this work is the formation of a large-scale magnetic field in a thin disk. One popular mechanism is assumed that there is initial weak large-scale magnetic field at the outer boundary of a thin disk, which can be magnified with the accretion of gas. However, the advection isn't effective for a standard thin disk because of the fast diffusion therein (Lubow et al. 1994). This problem can be solved by including the feedback of 

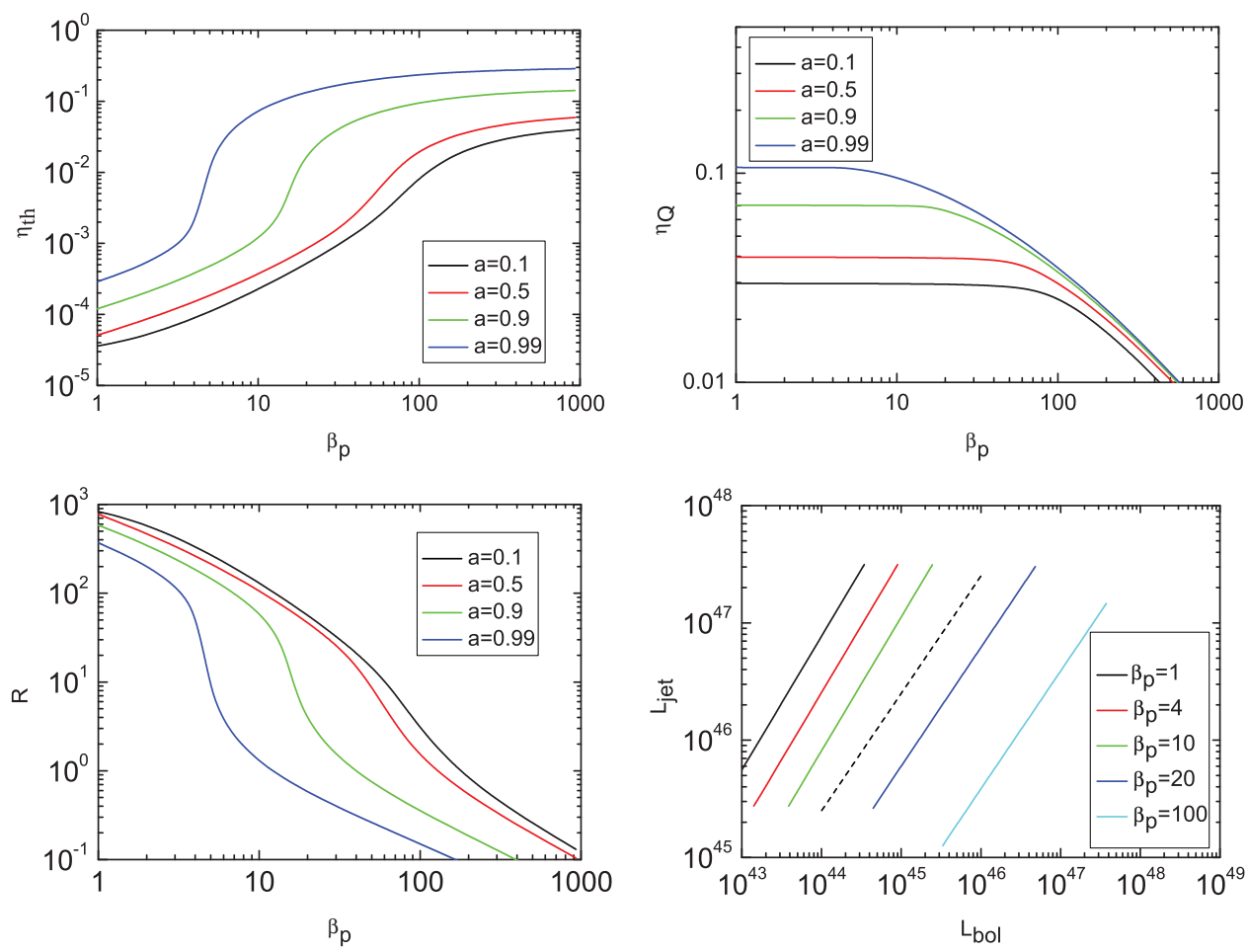

Figure 1. Panels (a), (b) and (c) are for the radiative efficiency $\eta_{\mathrm{th}}$, the jet production efficiency $\eta_{\mathrm{Q}}$ and the jet efficiency $R$ of a relativistic thin accretion disk with magnetically driven jets as functions of $\beta_{\mathrm{p}}$, respectively. And panel (d) represent the jet power as functions of bolometric luminosity for different $\beta_{\mathrm{p}}$ with $a=0.9$, where the black hole mass varies from $10^{8} M_{\odot}$ to $10^{10} M_{\odot}$. The dashed line corresponds to the observational jet efficiency lines $R=25$.

winds on accretion disk (Cao \& Spruit 2013; Li \& Begelman 2014). The formation of large scale magnetic field also depends on the initial strength and morphology of magnetic field in the MHD simulations (e.g., McKinney et al. 2012), which is still an open issue so far.

\section{Acknowledgements}

This work is supported by the NSFC (grants 11373056, 11233006).

\section{References}

Blandford, R. D. \& Payne, D. G. 1982, MNRAS, 199, 883

Blandford, R. D. \& Znajek, R. 1977, MNRAS, 179, 433

Cao, X., 2002, MNRAS, 332, 999

Cao, X., \& Spruit, H. C., 2013, ApJ, 765, 149

Fernandes C. A. C., Jarvis, M. J., Rawlings, S., et al., 2011, MNRAS, 411, 1909

Ghisellini, G. \& Celotti, A. 2001, A\& $A, 379$, L1

Li, S.-L., 2014, ApJ, 788, 71

Li, S.-L. \& Begelman, M. C., 2014, ApJ, 786, 6

Lubow, S. H., Papaloizou, J. C. B., \& Pringle, J. E., 1994, MNRAS, 267, 235

McKinney, J. C., Tchekhovskoy, A., \& Blandford, R. D., 2012, MNRAS, 423, 3083

Punsly, B., 2011, ApJ, 728, 17

Tchekhovskoy, A., Narayan, R., \& McKinney, J. C., 2011, MNRAS, 418, L79 